\section{Case report:} cobblestone

\title{
Haematospermia associated with malignant hypertension
}

\author{
Sumit Bhaduri, Vincent C Riley
}

Haematospermia is generally regarded as a benign, self limiting condition. Although no obvious cause is found in most cases, an uncommon but important predisposing factor is severe uncontrolled hypertension. We describe a case illustrating the need to routinely measure blood pressure in such instances.

(Sex Transm Inf 1999;75:200)

Keywords: haematospermia; hypertension

\section{Introduction}

Haematospermia is considered to be a benign disorder with published series either finding no cause or an inflammatory one. ${ }^{1-6}$ The prevalence of hypertension has been found to be $2.8 \%$ in three series of patients with haematospermia (177 cases). ${ }^{2}$ However, the association is generally with severe uncontrolled hypertension as the case below illustrates.

\section{Case report}

We report the case of a 36 year old man who initially presented to the genitourinary medicine clinic with a history of itching in the inguinal area of 3 weeks' duration. Coincidentally he mentioned that he had noticed blood in his ejaculate which he ascribed to minor trauma sustained when playing with his children. Clinical examination was suggestive of a bilateral tinea cruris. A routine blood pressure reading (as part of the clinic protocol for haematospermia) was 230/140. Urinanalysis showed blood and protein. Funduscopy revealed hard exudates and dot haemorrhages suggestive of a grade 3 hypertensive retinopathy. An urgent urea and electrolyte estimation showed the serum creatinine to be $237 \mathrm{mmol} / 1$. A chest $x$ ray was reported as normal and an electrocardiogram showed evidence of left ventricular hypertrophy. The patient was urgently referred to the renal unit where an ultrasound of the renal tract was suggestive of bilateral chronic parenchymal changes with no other focal abnormality. Renal biopsy showed evidence of glomerulosclerosis, with further studies indicating that the underlying pathology was suggestive of a IgA nephropathy. Lisinopril $10 \mathrm{mg}$ daily was commenced for his hypertension. On review, the patient did not report any recurrence of his haematospermia.

Department of

Genitourinary

Medicine, Leicester

Royal Infirmary,

Infirmary Square,

Leicester LE1 5WW

$S$ Bhaduri

V C Riley

Correspondence to:

Dr Sumit Bhaduri.

Accepted for publication 20 April 1999

\section{Discussion}

Haematospermia is regarded as benign and self limiting with resolution usually in less than a month. There are both local and general causes, the latter includes amyloidosis, cirrhosis, coagulopathies, and hypertension. ${ }^{1}$ Close et al however, have suggested that the association of haematospermia is with severe uncontrolled hypertension (as in this case) as opposed to hypertension per se. ${ }^{2}$ They based this hypothesis on comparison of a small series of patients who had both haematospermia and hypertension with matched control patients with just hypertension. The former group were found to have significantly higher systolic and diastolic blood pressures, higher serum creatinines, and higher left ventricular voltages based on ECG analysis. ${ }^{2}$ The aetiology of haematospermia has been ascribed to sudden emptying of the distended seminal vesicles during ejaculation causing haemorrhage from the ductal surface of the vesicles. ${ }^{1}$ Asymmetrical dilatation of one seminal vesicle as seen on transrectal prostatic ultrasonography, has been associated with severe hypertension. ${ }^{3}$ Other authors have suggested that the underlying process of haematospermia is related to varicosities of the posterior urethral veins, ${ }^{4}$ or pathology in the prostatic urethra. ${ }^{5}$

Occasionally, local pathology such as trauma, haemangioma of the glans penis, or prostatitis can be found. ${ }^{1}$ One series of patients has shown either an idiopathic cause $(38 \%)$ or an inflammatory cause $(32 \%)$ related to urethritis, prostatitis, seminal vesiculitis, or orchitis. ${ }^{6}$ Fletcher et al found an inflammatory cause in $80 \%$ of cases in their series (81 patients), particularly in those patients under the age of 30, and in addition, evidence of urological malignancy in only six out of the 81 cases. All cases in this latter group were over 40 years of age and had persistent haematospermia.

The case illustrates the need to check the blood pressure in any patient presenting with haematospermia as treatment may be required urgently.

We acknowledge the helpful contribution of Dr Graham Warwick, consultant physician in renal medicine, Leicester General Hospital, in the publication of this case report.

Murphy NJ.,Weiss BD. Hematospermia. Am Family Phys 1985;32:167-71. haemospermia and severe hypertension. Postgrad Med $\mathcal{F}$ 1991;67:157-8.

3 Weidner W, Jantos CH, Schumacher F, et al. Recurrent haemospermia - underlying urogenital anomalies and efficacy of imaging procedures. Br $\mathcal{f}$ Urol 1991;67:317-23.

4 Cattolica EV. Massive hemospermia: a new etiology and simplified treatment. F Urol 1980;124:151-2.

5 Stein AJ, Prioleau PG, Catalona WJ. Adenomatous polyps of the prostatic urethra, a cause of hematospermia. $\mathcal{F}$ Urol of the prostatic uret

6 Ross JC. Haemospermia. Practitioner 1969;202:59-62.

7 Fletcher MS, Herzberg Z, Pryor JP. Br f Urol 1981;53:66971. 